\title{
Acknowledgement to Reviewers of Religions in 2019
}

\section{Religions Editorial Office}

MDPI, St. Alban-Anlage 66, 4052 Basel, Switzerland

Published: 19 January 2020

The editorial team greatly appreciates the reviewers who have dedicated their considerable time and expertise to the journal's rigorous editorial process over the past 12 months, regardless of whether the papers are finally published or not. In 2019, a total of 646 papers were published in the journal, with a median time to first decision of 22.88 days and a median time from submission to publication of 46 days. The editors would like to express their sincere gratitude to the following reviewers for their generous contribution in 2019:

Abbas, Tahir

Abbott, Dena

Abdul Rahman, Noor Aisha

Abeysekara, Ananda

Abram, Anna

Adelakun, Abimbola A.

Aderibigbe, Simon

Adrian, John

Afridi, Mehnaz

Agudo Romeo, María Del Mar

Ahmad, Ahmad

Ahn, John

Aikin, Scott

Aitamurto, Kaarina

Akou, Heather

Al-Astewani, Amin

Alatas, Syed Farid

Alexander, Laura

Alexander, Michael S.

Ali, Jan

Ali, Muhamad

Allen, Douglas

Allen, Garrick

Allen, Garrick

Alley, Kelly

Almog, Yael

Almond, Philip

Alva, Reginald
Alvis, Jason

Ambrose, Glenn

Amin, Camron Michael

Amin, Hira

Ammon, Laura

Amore, Roy C.

Anderl, Christoph

Anderson, Bradford

Andolsen, Barbara

Andreopoulos, Andreas

Andrews, Susan

Andriessen, Karl

Anello, Giancarlo

Antes, Peter

Arigita, Elena

Arion, Alexandru-Corneliu

Aronowicz, Annette

Arruñada, Benito

Arthur, Shawn

Aslan, Rose S.

Assandri-Snoy, Friederike

Atzmon, Arnon

Aughterson, Kate

Aung-Thwin, Maitrii

Avni, Gideon

Ayubi, Zahra

Babie, Paul

Baffelli, Erica 


\begin{tabular}{|c|c|}
\hline Bagasra, Anisah & Berti, Daniela \\
\hline Bailén, Amparo Huertas & Bertolani, Barbara \\
\hline Bailey, Michael D. & Berzano, Luigi \\
\hline Bains, Satwinder Kaur & Beyerlein, Kraig \\
\hline Baker, Christopher & Bhogal, Balbinder S. \\
\hline Bakker, Hans & Bialecki, Jon \\
\hline Balmer, Brice & Bidegaín, Ana María \\
\hline Bañuls, Mónica Ruiz & Bienkowski, Piotr \\
\hline Bar, Alexandru & Bilan, Svitlana \\
\hline Barker, Adam & Block, Elizabeth Sweeny \\
\hline Barker, Eileen & Boaz, Danielle \\
\hline Barker, Wesley & Bock, Darrell L. \\
\hline Barnes, Bernadine & Bock, Gregory L. \\
\hline Barr, Nicolaas P. & Boender, Welmoet \\
\hline Bartelink, Brenda & Boggi, Flavio \\
\hline Bartkowski, John & Bonino, Stefano \\
\hline Basalamah, Salah & Boomkens, Cynthia \\
\hline Baskerville, Stephen & Booth, Paul \\
\hline Bass, Alden & Born, Christopher \\
\hline Bates, Larry W. & Bornemark, Jonna \\
\hline Batten, Alicia & Bosch, Míriam Díez \\
\hline Baugh, Amanda & Bosman, Frank \\
\hline Baum, Jacob M. & Bouchnick, Ram \\
\hline Baumann, Klaus & Bouma, Gary \\
\hline Baumgarth, William P. & Bounia, Alexandra \\
\hline Baumgartner, Christoph & Bourgel, Jonathan \\
\hline Bealer, Tracy & Bowd, Stephen \\
\hline Beall, Alyssa & Boyatzis, Chris \\
\hline Becci, Irene & Boyce-Tillman, June \\
\hline Beck, Guy & Bradley, John \\
\hline Beck, Herman & Bradley, Joseph \\
\hline Behl, Natasha & Branca, Paolo \\
\hline Belcher, Kimberly & Brandner, Tobias \\
\hline Bell, Christopher & Bratosin, Stefan \\
\hline Bell, Matthew & Bretzke, James \\
\hline Bell, Nicolas & Brock, Brian \\
\hline Bellomy, Donald & Bromber, Katrin \\
\hline Bengston, William & Brooks, Melanie \\
\hline Benton, Catherine & Brown, David \\
\hline Berberich, Christine & Brown, Jason Minton \\
\hline Bergmann, Emilie & Brown, Joseph \\
\hline Berry, Evan & Brown, Rachel Fulton \\
\hline Berthe, Jansen & Brown, Robert \\
\hline
\end{tabular}




\begin{tabular}{|c|c|}
\hline Brunk, Timothy & Chen, Huaiyu \\
\hline Brzozowski, Wojciech & Cheng, Wei-Yi \\
\hline Bsoul, Labeeb Ahmed & Cheruvallil-Contractor, Sariya \\
\hline Bubbio, Diego & Chesworth, John \\
\hline Bugslag, Jim & Cheu, Hoi \\
\hline Bühnemann, Gudrun & Chia, Philip \\
\hline Buhrman, William & Chitwood, Kenneth \\
\hline Bulag, Uradyn E. & Choi, Hyaeweol \\
\hline Burr, Brandon & Church Gibson, Pamela \\
\hline Bussanich, John & Ciković, Danijel \\
\hline Büssing, Arndt & Clark, Doug \\
\hline Buyze, David M. & Clegg, Cyndia Susan \\
\hline Calfano, Brian Robert & Clines, Gregory M. \\
\hline Calloway, Jamall & Clooney, Francis X. \\
\hline Camp, Cynthia Turner & Clorméus, Lewis Ampidu \\
\hline Campbell, Bolaji & Co Sy Su, Chadwick \\
\hline Cao, Nanlai & Cohen, Daniel \\
\hline Caplan, Jennifer & Cohen, Signe \\
\hline Caplan, Lionel & Cohen-Hanegbi, Naama \\
\hline Capper, Daniel & Coley, Jonathan \\
\hline Cappucci, John & Collet, Bruce \\
\hline Carey, Tamika L. & Collicutt, Joanna \\
\hline Cargill, Robert & Coman, Viorel \\
\hline Carile, Maria Cristina & Comeau, Katie \\
\hline Carl, John & Condon, Paul \\
\hline Carlbom, Aje & Cone, Pamela \\
\hline Carlson, Bronwyn & Considine, Craig M. \\
\hline Carnegie, Elizabeth & Conti, Fabrizio \\
\hline Carr, Jessica & Cook, Connie \\
\hline Carta, Giuseppe & Cook, Corey \\
\hline Cartledge, Mark & Correll, Mark \\
\hline Carvalho, Vinicius De & Cort, John \\
\hline Cary, Phillip & Cosgrove, Walker \\
\hline Cassidy, Kathryn & Cotter, Chris \\
\hline Cates, Diana & Cox, John \\
\hline Cavazos-Gonzalez, Gilberto & Coyle, Sean \\
\hline Cesari, Jocelyne & Craft, Christy D. Moran \\
\hline Chai, David & Craft, Jennifer Allen \\
\hline Chalmers, Matthew & Creel, Eileen \\
\hline Chan, Kwok Shing & Cristofori, Irene \\
\hline Chapple, Christopher Key & Crome, Andrew \\
\hline Charles Ryan, John & Crompton, Andrew \\
\hline CHEN, Hon-fai & Crook, Zeba \\
\hline
\end{tabular}


Croucher, Stephen

Crowley, Jocelyn

Cruz, Manuel

Cunningham, Christopher J. L.

Curran, John

Czaja, Olaf

Dabby, Dia

Dailey, Patricia

Dallh, Minlib

Dall'Olio, Guido

Dalton, Krista N.

Dalzell, Victoria M.

Dandelion, Pink

Daneshpour, Manijeh

Daniel, Dafydd Mills

Daniels, Denise

Darlington, Susan

Dart, Eric S.

Das Acevedo, Deepa

Davies, Oliver

Davis, Thomas J.

Davis, Tyler B.

Dawson, Stephen

De Haardt, Maaike

De Koning, Martijn

De Wildt, Kim

Death, Jodi

Debertolis, Paolo

Deeg, Max

DeHart, Paul

Delcore, Henry

Delcorno, Pietro

Delio, Ilia

DeLong-Bas, Natana J.

Deluga, Waldemar

Demmrich, Sarah

Denney, Andrew

Denny, Christopher

Denova, Rebecca

Desmond, Mary E.

Dessein, Bart

Dessi, Ugo

Destro, Robert A.
DeWitt, Lindsey

Dicken, Frank

Dickman, Nathan Eric

Dietrich, Jan

Dillinger, Johannes

Dinnell, Darry

Dippmann, Jeffrey

Discua, Allan

DiValerio, David

Dixon, Sandra

Dixon, Thomas

Dollahite, David

Dombrowski, Daniel

Donaldson, Brianne

Doukahan, Abigail

Dreff, Erik

Duckworth, Douglas

Duda-Seifert, Magdalena

Dugan, Katherine

Dumsday, Travis

Dundas, Paul

Duquette, Natasha

Easat-Daas, Amina

Edelmann, Jonathan

Edwards, Elise M.

Edwards, Mark

Eggen, Nora Sunniva

Eisenmann, Esti

Elbasani, Arolda

Eleff, Zev

Ellens, Jantina

Ellis, Robert

Elsässer, Sebastian

Elsner, Regina

Endresen, Cecilie

Engelmajer, Pascale

Epley, Steven

Epstein, Alek D.

Eric Daryl, Meyer

Erickson, Jacob J.

Erlich, Adi

Esler, Dylan

Essen, Johan Von 
Etengoff, Chana

Evolvi, Giulia

Ewart, Jacqueline

Faesen, Rob

Faggioli, Massimo

Fällman, Fredrik

Faries, Nathan C.

Farneth, Molly

Farwell, James W.

Faust, Avraham

Fauzia, Amelia

Fazel, Valerie

Featherstone, Daniel

Fech, Andrej

Fenech, Louis

Fernandes, Jason Keith

Fernandez, Christian

Ferrante, Marco

Ferré, John

Ferretter, Luke

Fine, Steven

Finger, Reta

Fisher, John W.

Fisk, Anna

Fisk, Rosemary

Fiskesjö, Magnus

Flam, Nancy

Flaskerud, Ingvild

Flesler, Daniela

Flügel, Peter

Flynt, Shannon R.

Fogg, Kevin W.

Foley, Edward

Fornerod, Anne

Francis, Leslie J.

Francisca, Cho

Franks, W. Paul

Frazier, Alison K.

Freeze, Gregory

Freiberger, Oliver

French, Katherine

Friesner, Nicholas Aaron

Fuller, Jason
Fuller, Robert

Gall, Andrew

Galloway, Charlotte

Galmiche, Florence

Ganany, Noga

Ganzel, Tova

Geaves, Ron

Gent, Bill

Gerber, Schalk Hendrik

Gheorghe, Huza

Ghosh, Abhishek

Gibler, Linda

Gijsberts, Marie-José H.E.

Gill, Anthony

Gill, Glen Robert

Gill, Harjant S.

Gill, Sam

Gill, Santokh

Gillespie, Ryan

Gilson, Caitlin

Gimbel, Steve

Giorgi, Alberta

Gish, Elizabeth

Glombik, Konrad

Göcke, Benedikt Paul

Goddard, Hugh

Gokani, Ravi

Gold, Malcolm

Goldstein, Warren S.

González, Rebeca Cristina López

Good, Anthony

Goodchild, Philip

Goodin, David K.

Gordon, Robert Edward

Gozdecka, Dorota Anna

Granoff, Phyllis

Grant, Airdre

Gravagnuolo, Antonia

Gray, David

Green, Roger

Greener, Aaron

Greenia, George D.

Greenough, Christopher 
Greiner, Alyson

Griera, Mar

Griffioen, Amber L.

Griffis, Rachel B.

Grimell, Jan

Grobien, Gifford

Groeneveld, Leanne

Gruen, Erich

Guillaume, Philippe

Guinness, Patrick

Guliciuc, Viorel

Gustafson, Hans

Guta, Mihretu

Guzmen-Carmeli, Shlomo

Gyatso, Janet

Hafsteinsson, Sigurjón Baldur

Halabi, Awad

Hamayon, Roberte

Hames, Harvey

Hamid, Sadek

Hamidi, Yalda

Hamilton, Jill

Hamm, Thomas

Hammer, Leonard

Hamoudi, Haider Ala

Hampton, Bryan

Han, Sam

Hancock, Rosemary

Hanel, Paul

Hankins, Davis

Hansen, Mette Halskov

Harding, James

Hardy, Ann

Harrison, Klisala

Hart, Jennifer

Hartung, Jan-Peter

Harvey, Paul

Hasan, Mubashar

Hashemi, Morteza

Hashemi, Nader

Hassenfeld, Ziva R.

Hatley, James

Hatley, Shaman
Hawkins, Billy

Hawthorne, Sian

Haynes, Jeffrey

Hayward, Richard D.

Healey, Kevin

Hedges, Paul Michael

Heern, Zackery M.

Hefty, Karl

Heifetz, Daniel

Heirman, Ann

Henderson, Andrea

Henderson, David

Hendrix, Scott

Herbert, David

Herman, Peter

Hernandez-Escobedo, Quetzalcoatl

Herrera Torres, Lucía

Herrero, Dolores

Herzfeld, Noreen

Heschel, Susannah

Heurtebise, Jean Yves

Hidalgo, Jacqueline

Hill, Chris

Hirono, Tatsushi

Hitzhusen, Gregory E.

Hjelm, Ingrid

Ho, Roger C.

Hoang, Linh N.

Hodge, Daniel

Hoffman, Louis

Hofmeyr, Isabel

Hogan, Cynthia

Hogue, Lawrence

Holmberg, Ingrid

Holmes-Tagchungdarpa, Amy

Holtorf, Cornelius

Holtschneider, Hannah

Hon, Tze-ki

Hood, Ralph

Horell, Harold

Horlacher, Rebekka

Horswell, Mike

Hovey, Joseph D. 
Howard, Cameron B. R.

$\mathrm{Hu}$, Hsiao-Lan

$\mathrm{Hu}$, Ying

Huang, WeiShan

Hue, Guan Thye

Hulsbosch, Marianne

Hunt, Jodi

Hunt, Jodi

Hunter, Erica C.D.

Hur, Nam-lin

Hussain, Amir

Hutch, Richard

Hutchinson, Dawn

Hwang, Merose

Hwang, Soon-Il

Hyland, Sabine

Hyland, William Patrick

Inbari, Motti

Inwood, Heather

Ireland, Daryl

Irvine, Richard

Irwin, Lee

Irwin, Msgr. Kevin W.

Isaacs, Alick

Jack, Alison

Jacobs, Steven

Jacobsen, Knut Axel

James, Anthony

James, Christine A.

Jasani, Rubina

Jasper, David

Ji, Chang-Ho

Jia, Jinhua

Jikeli, Gunther

Jillions, John

John, Tsz Pang

Johnson, Bryan

Johnson, Lee

Johnson, Rutherford

Johnson, Todd

Jones, Cassandra L.

Jones, Christopher D.

Jordan, Kevin
Jordan, Ryan

Jorgenson, Allen

Joseph, Celucien

Joubin, Rebecca

Jude, Sorana-Cristina

Juliette, Vuille

Jung, Jin-Heon

Jung, Jong Hyun

Kachru, Sonam

Kadivar, Mohsen

Kao, Chen-Yang

Kara, Seyfeddin

Kartveit, Magnar

Katunský, Dušan

Katz, Yaacov

Kavanagh, Chris

Kay, William

Kearney, Amanda

Kearney, Jonathan

Keenan, James

Keimer, Kyle

Kellenbach, Katharina

Kelman, Ari Y.

Kelsay, John

Kerley, Kent R.

Kettell, Steven

Kevern, Peter

Key, Andre

Killian, Mark

Kim, Halla

Kim, Heon

Kim, Myeongseok

Kim, Seong-Uk

Kimanen, Anuleena

King, Anna

King, Pamela Ebstyne

King, Richard

Kingsbury, Kate

Klaits, Frederick

Klemm, W.R.

Kohe, Geoffery

Kohn, Livia

Kojecky, Roger 
Koller, Aaron J.

Kolokytha, Chara

Könemann, Judith

Konzack, Lars

Kopania, Kamil

Kopár, Lilla

Kosky, Jeffrey

Krantz, David

Kraus, Paul

Kraybill, Donald B.

Krinks, Philip

Krop, Henri

Kruger, Matthew

Kuang, Lanlan

Kuczkiewicz-Fraś, Agnieszka

Kuha, Miia

Kuhle, Lene

Kujawa-Holbrook, Sheryl A.

Kuminova, Olga

Künkler, Mirjam

Kuo, Y. (Ya-pei)

Kuusisto, Arniika

Kwok, Wai Luen

Laamann, Lars

Ladouceur, Paul

LAI, Lufanna Ching-han

Lakitsch, Maximilian

Laliberté, André

Landrum, Brittany

Langenberg, Amy

Lanzarote-Fernández, María-Dolores

Lapidot, Elad

Large, William

Larsson, Göran

Last, Murray

Laugerud, Henning

Laumakis, Stephen J.

Laures-Gore, Jacqueline

Lavallee, Marc

Laven, Mary

Lavenia, Vincenzo

Lawrence, David

Lazarus, Suleman
Ledgerwood, Mikle D.

Lee, Becky $\mathrm{R}$

Lee, Joseph Tse-Hei

Lee, Song-Chong

Leibman, Laura

Leicht, Kevin T.

Leis-Peters, Annette

Leonard, Jeffery M.

Leth-Nissen, Karen

Levad, Amy

Levenson, Jon D.

Levey, Geoffrey

Levine, Daniel

Levinson, Eyal

Levy, Denise

Levy, Dore J.

Lewis, Christopher Alan

Lewis, Jonathan

Lewis, Philip

Li, Ji

Liberatore, Giulia

Lidke, Jeffrey

Lieber, Laura S.

Lienesch, Michael

Lin, Hsi-Chuan

Lindholm, Charles

Litvak, Meir

Liu, Jifeng

Liu, Peng

Liu, Xiaoqing

Liutikas, Darius

Llewellyn, J. E.

Lloyd, Vincent

Lo Giudice, Alessio

Lo, Yuet Keung

Loader, William

Lochtefeld, James

Locklin, Reid B.

Long, Brad S.

Long, Jeffery D.

Long, Kenneth J.

Loo, Tze M.

Lopez Villanueva, Cristina 


\author{
López-López, Daniel \\ Lovat, Terence \\ Lovat, Terry \\ Lovins, Christopher \\ Loy, Hui-chieh \\ Luo, Liang \\ Lupton, Julia \\ Lynn, Richard John \\ Mabon, Simon \\ Macdonald, Sean \\ MacInnis, John \\ Madathummuriyil, Sebastian \\ Mahdavi, Mojtaba \\ Majeed, Debra \\ Makovetsky, Lea \\ Maleuvre, Didier \\ Malisa, Mark \\ Marbaniang, Domenic \\ Maria Chambers, Claire \\ Marienberg, Evyatar \\ Markides, Kyriacos \\ Marks, Loren \\ Marquez, Xavier \\ Marshall, Alison R. \\ Marti R. Jewell, D.Min. \\ Martin, Kameelah L. \\ Mashiach, Amir \\ Mason, David \\ Master, Daniel \\ Matemba, Yonah \\ Matos Silva, Fátima \\ Maud, Jovan \\ Maxwell-Stuart, Peter \\ May, Matthew \\ Mayse, Ariel Evan \\ McAdam, Marika \\ McBrayer, Justin \\ McBride, Richard D. \\ McCarron, Richard E. \\ McCarthy, Susan \\ McClelland, Gwyn \\ McDaniel, June \\ McDaniel, Justin
}

McDermott, Rachel

McDowell, Liz

McDuffie, David C.

McEwan, Cameron

McGinnis, Scott

McGrady, Andrew G.

McGrath, William

McKeogh, Colm

McLaughlin, Eleanor

McMichael, Steven

McNair, Bruce

Mcphie, Jamie

Medine, Carolyn

Meek, Allen

Meier, David

Meier, Sam Arthur

Mell, Julie

Menon, Dilip

Mercadante, Linda

Mercer, Calvin

Metress, Christopher

Mews, Constant

Meyer, Andrew

Meyers, Carol L.

Michaelson, Jay

Mickey, Sam

Miedema, Siebren

Mikeshin, Igor

Milani, Milad

Miller, Caroline

Miller, Gavin

Miller, James

Miller, Nicholas P.

Miller, Timothy S.

Milofsky, Carl

Mislin, David

Mitchem, Stephanie Y.

Mitrofanova, Anastasia

Moeller, Hans-Georg

Mohamed, Mohamed Mosaad Abdelaziz

Mokhoathi, Joel

Monnot, Christophe

Moody, Ivan 


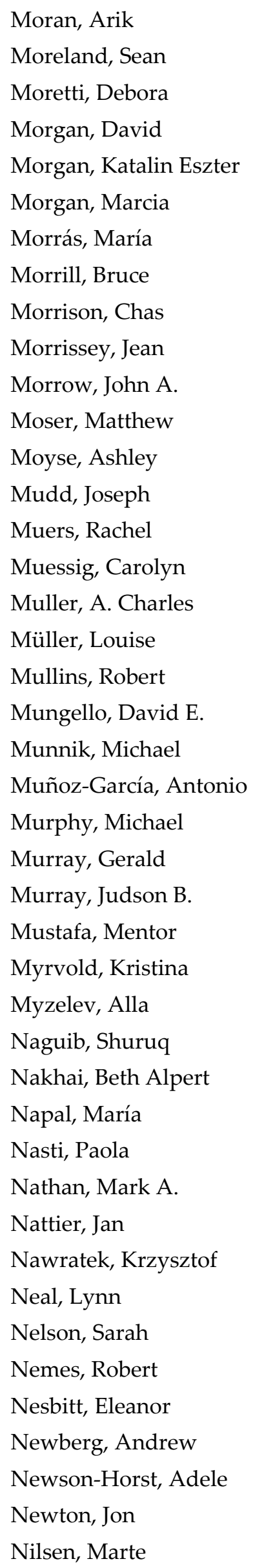

Nixon, Graeme

Nodet, Étienne

Noll, Mark

Nordvig, Mathias

Norris, Kristopher

Norton, Allison

Norton, Michael

Noseworthy, William B.

Nourse, Jennifer

Novak, Maximillian E.

Nugteren, Albertina (Tineke)

Nussmeier, Anthony

O'Donnell, Kate

Obadia, Lionel

Obrock, Luther

Ochs, Peter

Oden, Patrick

Odorisio, David

Okholm, Dennis

Oleszkiewicz-Peralba, Malgorzata

Olive, Sarah

Olsen, Daniel

O'Meara, Simon

Omry, Keren

O'Neil, Mary

O'Neill, Mark

Onians, John

Ooms, Julie

Oppy, Graham

Ornella, Alexander Darius

Osborne, Monica

OSEI, JOSEPH

Ospino, Hosffman

O'Sullivan, Michael E.

Othman, Enaya

Owens, Bob

Paal, Piret

Pace, Enzo

Paipais, Vassilios

Palmer, Clive

Palmer, David

Parish, Helen

Park, Thomas K. 
Parrillo, Vincent N.

Patel, Vibhuti N.

Patrick, Margie

Pattison, George

Patton, Pamela

Pauly, Robert J.

Payne, Richard

Pedersen, Anders Klostergaard

Pelayo, Edgar Zavala

Pena, Milagros

Pennington, Brian K.

Pennington, Madeleine

Pentaris, Panagiotis

Pepinsky, Thomas

Perlmutter, Haim

Perselis, Emmanouil

Peters, Greg

Petrone, Michele

Pettid, Michael J.

Pettit, Jonathan

Petts, Richard J.

Phillips, Amanda

Phillips, Jeff

Phillips, Thomas E.

Piccinin, Antonella

Pickel, Gert

Pieri, Zacharias

Pihlaja, Stephen

Pihlström, Sami

Pintér, Károly

Plate, Brent

Poché, Justin D.

Poleschuk, Irina

Polgar, Nenad

Poljarevic, Emin

Pommaret, Francoise

Porobić, Selma

Porpora, Douglas

Portier-Young, Anathea

Porumb, Razvan

Potts, Grant

Powers, Paul

Prevelakis, Nicolas
Prideaux, Mel

Punathil, Salah

Putter, Ad

Qadir, Ali

Quli, Natalie

Radice, Thomas

Raffety, Erin

Rahman, Osmud

Rajpurohit, Dalpat

Ramírez, Jesse

Ramsay, Nancy

Rana, Meenal

Rane, Halim

Ranjan, Rahul

Rausch, Franklin

Ravven, Heidi

Rech, Giovanna

Reed, Esther D.

Reed, Pamela G.

Rees, Dafydd Huw

Rees, John

Reinkowski, Maurus

Repo-Saeed, Nora

Richardson, Alasdair

Riches, Tanya

Ricucci, Roberta

Rinderknecht, Jakob

Rinehart, Robin

Ritari, Katja

Rivera, Joseph

Robbins, Brent Dean

Robbins, Mandy

Robert, William

Roberts, Joanne E.

Roberts, Stephen

Roche Cárcel, Juan Antonio

Rodrigues, Donizete

Rodriguez, Agustin Martin G.

Rogers Healey, Robynne

Rolston, Holmes

Rončáková, Terézia

Rosario-Rodriguez, Ruben

Rota, Michael W. 
Rots, Aike Peter

Rountree, Kathryn

Roussos, Sotiris

Rouzati, Nasrin

Royalty, Robert M.

Ruane, Nicole

Rudnick, Abraham

Ruggles, Steven

Ruhmkorff, Samuel

Rumbold, Bruce

Rundle, Steven

Sahin, Abdullah

Saiz Galdós, Jesús

Sajid, Nida

Salvant, Shawn

Sammons, David

Samson, Maxim

Samuel, Geoffrey

Sánchez Sánchez, Laura Del Carmen

Sandikci, Özlem

Sansom, Dennis

Santos Marinas, Enrique

Santos, Xosé

Sarris, Peter

Sasson, Vanessa

Scarborough, Connie

Schedneck, Brooke

Scheid, Anna Floerke

Scheitle, Chris

Schliesser, Christine

Schmidt, Christoph

Schmidt, Cindy

Schofer, Jonathan

Scholes, Jeff

Schonebaum, Andrew

Schorch, Stefan

Schreiber, Monika

Schumm, Walter R.

Schwartz, Yosef

Schwarz-Friesel, Monika

Schweig, Graham

Scott-Baumann, Alison

Sengupta, Anindita
Seniguer, Haoues

Serafini, Gianluca

Seymour, Jack L.

Shahar, Galili

Shakeel, M. Danish

Shariff, Shaheen

Sharma, Gitima

Shaw, Sarah

Sheikh, Faraz

Shemesh, Abraham Ofir

Sherma, Rita

Sherri, Brown

Sherwood, Tom

Shi, Xia

Shi-Kupfer, Kristin

Shirazi, Faegheh

Shnirelman, Victor

Shoemaker, Terry

Show, Ying Ruo

Shuger, Dale

Simmons, Caleb

Simon, Thompson

Simpson, Ian

Singh, Amit

Singh, Harpreet

Singh, Pashaura

Sinkevic, Ida

Sjö, Sofia

Skaptadóttir, Unnur Dís

Skinnebach, Laura Katrine

Smith, Darron

Smith, Ei Phyu

Smith, Eric C.

Smith, Norman

Smith, Sharon

Smyer Yu, Dan

Snook, Jennifer

Soliman, Asmaa

South, Robbie M.

Souza, Carlos Frederico Barboza De

Spickard, James

Sponsel, Leslie E.

Squiers, Anthony 


\author{
Stadel, Christian \\ Stadtner, Donald \\ Starr, Chloë \\ Stecz, Patryk \\ Steil, Carlos \\ Steinhardt, Nancy Shatzman \\ Stensvold, Anne \\ Stewart, James \\ Stirr, Anna \\ Stjerna, Kirsi \\ Stoeckl, Kristina \\ Strandberg, Hugo \\ Strong, David \\ Stuart, Elizabeth \\ Studzinski, Raymond \\ $\mathrm{Su}$, Yuxiao \\ Sukhu, Gopal \\ Sulistiyanto, Priyambudi \\ Sullivan-González, Douglass \\ Summers, Jesse \\ Sundaram, Dheepa \\ Sureda, Marc \\ Sutton, Timothy \\ Svob, Connie \\ Swanson, Joel \\ Swanson, Paul \\ Swick, Danielle \\ Sygit, Katarzyna \\ Tacik, Przemysław \\ Tam, Lui \\ Taneti, James Elisha \\ Tappan, Robert \\ Tarocco, Francesca \\ Tartakoff, Paola \\ Taylor, Lynne \\ Tervanotko, Hanna \\ Thatcher, Adrian \\ Theodor, Ithamar \\ Thomas, Scott \\ Thomases, Drew \\ Thompson, Glen L. \\ Thrall, James H. \\ Tiaynen-Qadir, Tatiana
}

Ticciati, Susannah

Tiffany, Grace C.

Todd, Andrew

Tolar, Tanja

Tollerton, David

Toroczkai, Ciprian Iulian

Traina, Cristina L. H.

Trainor, Michael

Travagnin, Stefania

Trice, Michael Reid

Trompf, Garry

Tsitselikis, Konstantinos

Tsuria, Ruth

$\mathrm{Tu}$, Yichao

Tucker Edmonds, Joseph L.

Tuell, Steve

Tull, Herman

Turcan, Nicolae

Turek, Lauren

Turk, Elizabeth Hunter

Tyson, Craig W.

Ukpokodu, Peter

Underdahl, Louise

Unsworth, Amy

Urban, David V.

Uzukwu, Elochukwu

Vallely, Anne

Valutyte, Regina

Van Den Akker, C.M.

Van Den Hemel, Ernst

Van Der Schyff, Gerhard

Van Doore, Kate

Van Doorn-Harder, Nelly

Van Engeland, Anicee

Van Es, Margreet

Van Gulik, Léon

Van Ommen, Léon

Van Overmeire, Ben

Van Raemdonck, An

Vanderputten, Steven

Vanzan, Anna

Vargas OBryan, Ivette

Veracini, Lorenzo 
Verhoef, Anné Hendrik

Vermeer, Paul

Vermurlen, Brad

Vicelja, Marina

Vicini, Andrea

Villadsen, Kaspar

Visser, Adriaan $\mathrm{Ph}$

Vlahos, Margaretha

Von Collani, Claudia

Vose, Steven

Voulgaropoulou, Margarita

Wadley, Susan Snow

Waite, Gary

Walker, Anthony

Walker, Marvin

Wall, John

Wallace, Vesna

Waller, Steven

Wallwork, Ernest

Walser, Joseph G.

Walton, Martin N.

Wang, Xiuhua

Ward, Kate

Ward, Kevin

Weathers, Elizabeth

Weaver-Zercher, David L.

Weber, Charles

Webman, Esther

Weed, Ronald

Weidner, Chad

Weismann, Itzchak

Weller, Robert

Welter, Albert

Westendorp, Mariske

Wetmore, Kevin

Wetterberg, Ola

Whalen, Benedict

Wheeler, Kayla

Whitaker, Robyn

White, Chris

White, Grant S.

White, Richard J.

Whitehead, Amy
Whitehead, Andrew

Wight, Linda

Williams, Joshua T.B.

Williams, Rachel

Williamson, Paul

Willows, Adam

Wills, Anne Blue

Wilmington, David

Wilmoth, Joe

Wilsey, John D.

Wilson, Joseph A

Wilson, Tom

Winings, Kathy

Winright, Tobias

Wittekind, Folkart

Wolffram, Heather

Wollina, Torsten

Wong, Man Kong Timothy

Wood, Ben

Woodman, Justin

Worcester, Thomas

Worley, Taylor

Wright, Dale

Wright, Elina

Wright, Robin M.

Wu, Emily

$\mathrm{Wu}$, John

$\mathrm{Xu}$, Xiaohe

$X u$, Zhiqiu

Yanchevskaya, Nataliya

Yasmeen, Samina

Yazdani, Mina

Yeager, Jonathan

Yendell, Alexander

Yengde, Suraj

Yesudhasan, Thomas

Yigal, Levin

Yoon, SungMan

Young, Jonathan

Young, Stuart

Yu, Chi-Ying

Yu, Shuishan

Zaborskis, Apolinaras 
Zachman, Randall C.

Zarrabi-Zadeh, Saeed

Zeller, Benjamin

Zilio-Grandi, Ida

Ziporyn, Brook
Zuhur, Sherifa

Zurlo, Gina

Zurn, Perry

Zürn, Tobias Benedikt

Zwingmann, Christian

(C) 2020 by the author. Licensee MDPI, Basel, Switzerland. This article is an open access article distributed under the terms and conditions of the Creative Commons Attribution (CC BY) license (http://creativecommons.org/licenses/by/4.0/). 